\title{
The Potential Geographical Distribution of Locusta migratoria tibetensis Chen (Orthoptera: Acrididae) in Qinghai-Tibet Plateau*
}

\author{
Xiongbing $\mathrm{Tu}^{1,2}$, Zhihong $\mathrm{Li}^{1,{ }^{* *}}$, Zehua Zhang ${ }^{2, *}$, Zhigang $\mathrm{Wu}^{1}$, \\ Wenlong $\mathrm{Ni}^{1}$, Liao $\mathrm{Fu}^{1}$, and Yasen $\mathrm{Shali}^{2}$ \\ ${ }^{1}$ Department of Entomology, College of Agriculture and Biotechnology, \\ China Agricultural University, Beijing 100193, China \\ ${ }^{2}$ Key Laboratory for Biological Control of Ministry of Agriculture, \\ Institute of Plant Protection, Chinese Academy of Agricultural Sciences, Beijing, 100193, China \\ txb1208@163.com, lizh@cau.edu.cn, lgbcc@263.net
}

\begin{abstract}
Locusta migratoria tibetensis Chen (Orthoptera: Acrididae) is one of the most harmful species of Acrididae. It causes extensive damage to gramineous crops. The climate mapping program, CLIMEX 3.0, and the GIS software, ArcGIS 9.3, were used to model the current and future potential geographical distribution of $L . m$. tibetensis. The model predicts that, under current climatic conditions, L. m. tibetensis will be able to establish itself throughout much of Qinghai-Tibet Plateau in China, including Qinghai, Tibet and Sichuan province. A total of $94.23 \%$ of Qinghai-Tibet Plateau's land mass or $2.22 \times 10^{6}$ $\mathrm{km}^{2}$, is climatically suitable under current climatic conditions. In this study, the potential distribution of $L$. m. tibetensis under future climate scenarios was projected and quantified climatically suitable areas. In Qinghai-Tibet Plateau, the total suitable climate land mass was increased by $0.16 \times 10^{6} \mathrm{~km}^{2}$ or $0.70 \%$ until 2100. However, compared to 2020 and 2050, the suitable land mass was decreased by $0.47 \times 10^{6} \mathrm{~km}^{2}$ and $0.17 \times 10^{6} \mathrm{~km}^{2}$. The most optimal area would increase $0.16 \times 10^{6} \mathrm{~km}^{2}$ until 2100, the most of Qinghai-Tibet Plateau would be expected to offer optimal climatic conditions for the possible spread and establishment of the pest. The analysis of this information indicated a high degree of accuracy for the CLIMEX model. The significant increases in the potential distribution of $L$. m. tibetensis projected under the climate change scenarios considered in this study suggest that biosecurity authorities should consider the effects of climate change when undertaking pest risk assessments.
\end{abstract}

Keywords: Locusta migratoria tibetensis Chen, CLIMEX, potential geographic distribution, ArcGIS, climate change.

\footnotetext{
* This study received financial supports from the Special Fund for Agro-scientific Research in the Public Interest (201003079).

** Corresponding author, China Agricultural University and Chinese Academy of Agricultural Sciences contributed equally to this paper.
} 


\section{Introduction}

Locusta migratoria tibetensis Chen is one of the most harmful pests and causes large amounts of damage to gramineous crops. Since 1990s, the occurrence of L. m. tibetensis has showed a rising trend. Especially in 2003 to 2006, there were 1000 to 3000 individuals per $\mathrm{m}^{2}$ along the valley areas of Jinsha River, Yarlung Zangbo River and Yalong River, which had threaten the production of highland barley and grass [1-2].

The distribution area of L. m. tibetensis is only in China at present, including northern of Qinghai, Tibet and west of Sichuan province[3]. Studies showed that there was a significant correlation between increasing of potential distribution area and global warming. The annual surface temperature increased at the speed of $0.0301^{\circ} \mathrm{C}$, while the potential distribution area increased $504.38 \mathrm{~km}^{2}$ per year. This result indicated that a little increment in temperature would bring a great extension in distribution of L. m. tibetensis[2].

The CLIMEX model has been used to describe the potential distribution of some pests, such as Ceratitis capitata (Wiedemann) [4-5], Bactrocera tryoni(Froggatt) [6-7], Carpomya vesuviana Costa[8], B. tsuneonis(Miyake)[9], B. scutellata (Hendel)[10], Toxotrypana curvicauda Gerstaecker[11], B. zonata(Saunders)[12], Melanoplus sanguinipes (Fabricius) [13]. This model has also previously been used to predict the effects of climate change on species' potential distributions using either a regional global climate model [14] or synthetic climates[15-16]. Species examined using this approach include B. dorsalis (Handel)[17], Melaleuca quinquenervia[18] and Melanoplus sanguinipes (Fabricius)[13]. To know the potential distribution of the L. $m$. tibetensis under climate change assumptions due to potential climate change impact, the CLIMEX 3.0 model was used to assess the response of L. m. tibetensis to climate and to make predictions for the 2100 s.

\section{Materials and Methods}

\subsection{Overview of the CLIMEX Model}

CLIMEX is a dynamic model[19] that integrates the weekly responses of a population to climate using a series of annual indices. The growth and stress indices are calculated weekly and then combined into an overall annual index of climatic suitability, the Ecoclimatic Index (EI). This index is expressed on a scale of 0 to 100 . Some suggested guidelines are provided[20-21] based on the EI. An EI of 0.00 indicates that the climate is unsuitable for the species and that the species cannot persist in an area under average environmental conditions. An EI of 0.01-9.99 indicates marginal conditions, an EI of 10.00-19.99 indicates suitable conditions, and an EI of 20+ indicates optimal conditions. An EI of 100 indicates that conditions are perfect throughout the year. However, few environments are sufficiently stable to provide perfect habitat all year[17]. 
To visualize the results, the CLIMEX output was 'loose-coupled'[16] to a geographical information system (ArcGIS). This system was purchased from ESRI to create thematic maps. ArcGIS was also used to make projections of the land areas that would have a suitable climate for the pest species.

\subsection{Meteorological Databases and Climate Change}

Two climate databases were used in this modeling exercise. By default, CLIMEX uses 30-year averages of climate data to estimate climatic suitability. The CLIMEX standard meteorological dataset was first used to create an initial fit. This dataset accompanied CLIMEX version 3.0 and consists of 30-year averages from 1961 to 1990 for an irregularly spaced set of 2500 climate stations. Subsequently, a regular girded dataset of the normal climate for the same period TYN SC 2.0 (http://www.cru.uea.ac.uk/ timm/grid/TYN_SC_2_0.html)[22] was used to fine-tune the parameter fit. The dataset consisted of 67,420 points spaced on a $0.5^{\circ}$ latitude $\times 0.5^{\circ}$ longitude regular grid for significant land areas worldwide.

The future average annual temperature will increase $1.3-2.1{ }^{\circ} \mathrm{C}, 2.3-3.3^{\circ} \mathrm{C}, 3.9-6^{\circ} \mathrm{C}$ until 2020, 2050, 2100 respectively. While the future annual mean precipitation will increase $2-3 \%, 5-7 \%, 11-17 \%$ until 2020, 2050, 2100 respectively in China[17,23]. In this study, we use the average data of temperature and annual mean precipitation for the potential geographical distribution analysis in the future.

\subsection{Fitting CLIMEX Parameters}

To fit the CLIMEX model for L. m. tibetensis, the parameters were manually and iteratively adjusted until the simulated geographical distribution, as estimated by the EI values, coincided with the species' known native distribution. Parameters used in the CLIMEX model are presented in table 1.

\section{Temperature Index}

In this paper, the minimum temperature for development (DV0) was set at $14.6^{\circ} \mathrm{C}$ ( $\mathrm{Li}$ et $a l ., 2007)$. The lower and upper optimum temperatures for L. m. tibetensis populations were set at $15-28^{\circ} \mathrm{C}[24]$. So the lower and upper temperature optima (DV1 and DV2) were set at 15 and $28^{\circ} \mathrm{C}$, respectively[24-25]. As a result of the species is established in Tibet, and the day average temperature is no more than $32^{\circ} \mathrm{C}$ (http://cdc.cma.gov.cn/), so the upper threshold temperature (DV3) was set at $32^{\circ} \mathrm{C}$.

\section{Moisture index}

In commonly, Locust like desiccated environment for embryonic development stage. They survive well when the soil moisture in May at 0.03-0.3[26]. However, we have found that the soil moisture can be reach to 0.40 in October, this would be related with there was a long snow-covered time in Qinghai-Tibet Plateau. So in this paper, the 
lower soil moisture limit for development (SM0) was set to 0.02, the lower and upper limits for optimal growth (SM1 and SM2) were set to 0.03 and 0.5 respectively[25,27]. According to one year soil moisture changes of Tibet, the upper soil moisture limit for development, SM3, was set to 0.7 (http://cdc.cma.gov.cn/).

Table 1. Parameters used in the CLIMEX model for the L. m. tibetensis

\begin{tabular}{ll}
\hline Parameter & Value \\
\hline DV0 Lower threshold temperature & 14.60 \\
DV1 Lower optimum temperature & 15.00 \\
DV2 Upper optimum temperature & 28.00 \\
DV3 Upper threshold temperature & 32.00 \\
SM0 Lower threshold of soil moisture & 0.02 \\
SM1 Lower limit of optimum soil moisture & 0.03 \\
SM2 Upper limit of optimum soil moisture & 0.50 \\
SM3 Upper threshold of soil moisture & 0.70 \\
DPD0 Diapause induction day length & 11 \\
DPT0 Diapause induction temperature (average weekly minimum) & 11.0 \\
DPD1 Diapause termination temperature (average weekly minimum) & 3.0 \\
DPD Diapause development days & 120 \\
DPSW Summer or winter diapause ('0' for winter diapause, '1'for summer & \\
diapause) & \\
TTCS Cold stress temperature threshold & 0 \\
THCS Cold stress accumulation rate & -17.00 \\
TTHS Heat stress temperature threshold & -0.0003 \\
THHS Heat stress accumulation rate & 33.00 \\
SMDS Dry stress soil moisture threshold & 0.005 \\
HDS Dry stress accumulation rate & 0.002 \\
SMWS Wet stress soil moisture threshold & -0.003 \\
HWS Wet stress accumulation rate & 0.70 \\
\hline & 0.001 \\
\hline
\end{tabular}

\section{Diapause Parameter}

Diapause is an important biology characteristic for locust to endure bad environment. In this paper, we take Melanoplus sanguinipes (Fabricius) for setting diapause parameter of L. m. tibetensis[13]. Compared to Melanoplus sanguinipes, the diapause day length and development days of L. m. tibetensis are very close to Melanoplus sanguinipes (http://cdc.cma.gov.cn/), also L. m. tibetensis is winter diapauses[26]. 
However, there was no related report about diapause induction and termination temperatures.

\section{Stress Parameter}

All stress parameters were adjusted by the real distributions in China, to ensure that the predictions of areas suitable for the insect were consistent with the insect's current absence.

\subsection{Estimating the Size of Land Areas of Suitable Climate Projected by the CLIMEX Model}

The land areas projected to have a climate suitable under current and future conditions for L. m. tibetensis (EI>0) were quantified for Qinghai-Tibet Plateau (table 2).

Table 2. Projected area that is climatically suitable (EI>0) for $L$. $m$. tibetensis under the current climate, expressed as an area $\left(10^{6} \mathrm{~km}^{2}\right)$ and as a percentage of the total land of Qinghai-Tibet Plateau, and the percentage changes in Qinghai-Tibet Plateau for 2020, 2050 and 2100 in comparison with those obtained under the current climate. The climate model was run for the future climate changes in China[23].

\begin{tabular}{ccccc}
\hline & $\begin{array}{c}\text { Area with EI }>0 \text { under } \\
\text { current climate }\end{array}$ & \multicolumn{2}{c}{$\begin{array}{c}\text { Mean changes in areas with EI>0 under future } \\
\text { climate in comparison with those obtained }\end{array}$} \\
\cline { 3 - 5 } & 2.22 & -0.47 & -0.17 & 0.16 \\
\hline $\begin{array}{c}\text { Total area } \\
\left(10^{6} \mathrm{~km}^{2}\right)\end{array}$ & 94.23 & -1.99 & -0.72 & 0.70 \\
\hline total land area & & & 2050 & 2100 \\
\hline
\end{tabular}

\section{$3 \quad$ Results}

\subsection{The Potential Distribution of L. m. Tibetensis under Current Climatic Conditions}

The projections using the model suggest that under current climatic conditions, $L$. $m$. tibetensis can potentially establish itself to the Qinghai-Tibet Plateau (fig. 1, a). The results of the analysis indicate that Qinghai-Tibet Plateau would be expected to offer optimal climatic conditions for the possible spread and establishment of the pest. After CLIMEX modeling, the extent of the land area that is climatically suitable for $L$. $m$. tibetensis under the current climate is quantified by ArcGIS in table 2. A total of $94.23 \%$ of Qinghai-Tibet Plateau's land mass or $2.22 \times 10^{6} \mathrm{~km}^{2}$, is climatically suitable. 


\subsection{The Potential Distribution of $\mathbf{L}$. $\mathbf{m}$. Tibetensis under Future Climate Scenarios}

In this study, the potential distribution of L. m. tibetensis under future climate scenarios was projected and quantified climatically suitable areas. In Qinghai-Tibet Plateau, the total suitable climate land mass was increased by $0.16 \times 10^{6} \mathrm{~km}^{2}$ or $0.70 \%$ until 2100 (table 2). However, compared to 2020 (fig.1, b) and 2050 (fig.1, c), the suitable land mass was decreased by $0.47 \times 10^{6} \mathrm{~km}^{2}$ and $0.17 \times 10^{6} \mathrm{~km}^{2}$ (table 2). The most optimal distribution area (EI: 20.00+) would increase $0.16 \times 10^{6} \mathrm{~km}^{2}$ until 2100 (fig.1, d).
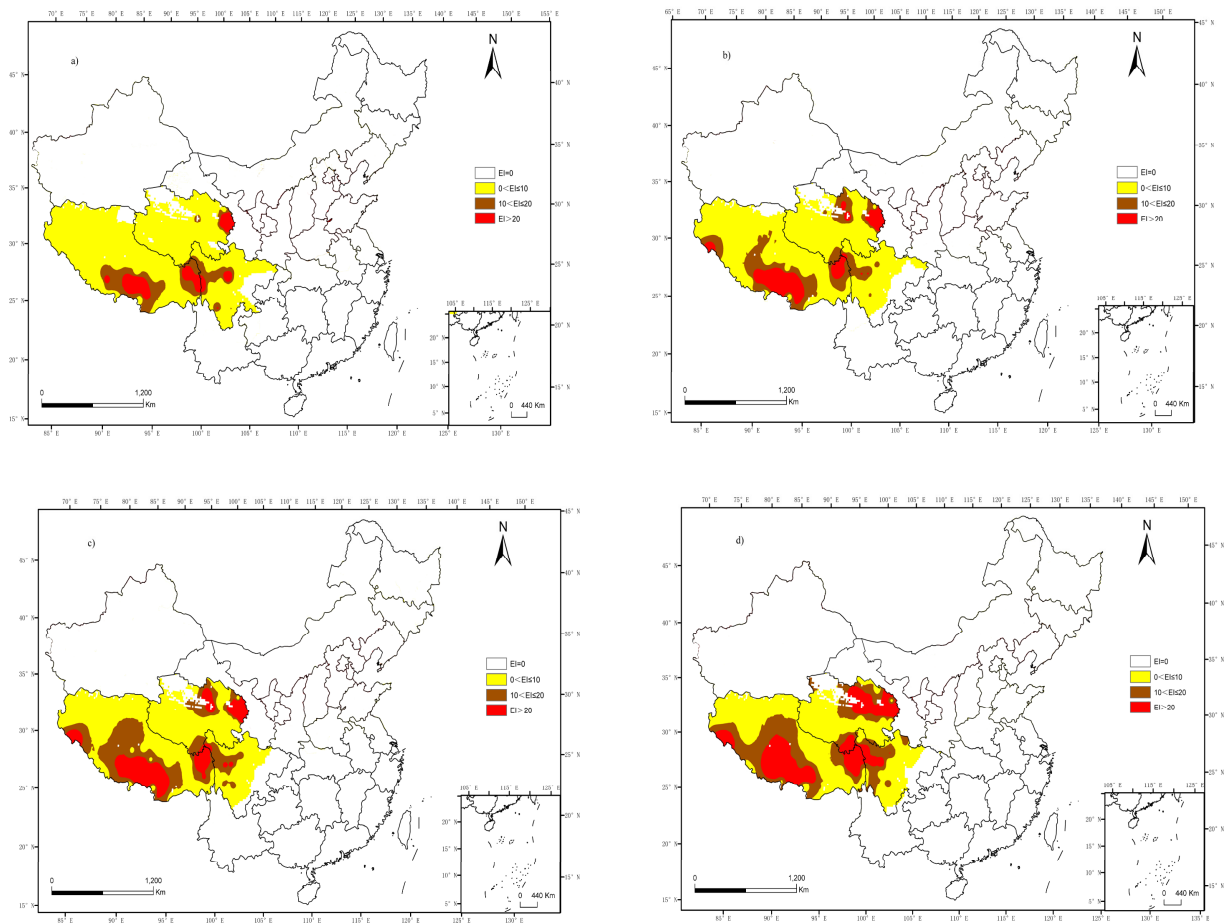

Fig. 1. Climatic suitability (EI) for the locust in the current and future climate scenarios projected using CLIMEX ${ }^{\mathrm{TM}}$. a)Qinghai-Tibet Plateau, current climate; b)Qinghai-Tibet Plateau, 2020; c)Qinghai-Tibet Plateau, 2050; d)Qinghai-Tibet Plateau, 2100. $\square$, unsuitable (0.00); $\square$, marginal (0.01-10); $\square$, suitable (10-20); $\square$, optimal (20.00+).

\section{Discussion}

L. m. tibetensis as the independent subspecies which has been nominated by Chen[3]. The taxonomy of $L$. $m$. tibetensis has been studied recently; Zhang et al.[28-29] had illustrated the potential importance of phylogeographical history in shaping the divergence and distribution patterns of $L$. m. tibetensis and other subspecies, he thought 
that L. m. tibetensis had independent genetic evidence for strong population substructure, as a result of geographical blocking for there was no gene flow between the inner and outside of Qinghai-Tibet Plateau. This is the reason for the current distribution of L. m. tibetensis, and we only predicted the potential distribution in Qinghai-Tibet Plateau in this paper.

The results of this study indicate that L. m. tibetensis is expected to be able to establish itself under temperate zones. Such conditions are to be found in most of Qinghai-Tibet Plateau. Moreover, the results suggested that as climate changes, the potential distribution of L. m. tibetensis would expand into cold regions, as it would expand into northward of the Plateau.

We have known that L. m. tibetensis is only in Qinghai-Tibet Plateau at present[3]. Studies showed that a little increment in temperature would bring great extension in distribution of L. m. tibetensis[2]. This study presented an example of influence of global warming on plateau ecology. So how climatic changes influence the potential distribution of insect in Qinghai-Tibet Plateau will need more attentions[30-31].

Potential distributions cannot be predicted based on climate alone. There is also a need to consider dispersal and species interactions, such as host availability. For example, mangoes, Mangifera indica Linn, are distributed in over 70 countries, more than $90 \%$ are grown in Asia (India, Pakistan, Bangladesh, Myanmar, Malaysia, China, Thailand, etc.), while Tanzania, Zaire, Brazil, Mexico, the United States, etc., also cultivate mangoes[32]. Competition and the effects of natural enemies[33] should also be considerations. Moreover, the limiting factors considered to affect the geographical distributions of pests should include soil type, geographical features, natural and geographical barriers and human activities. For example, the host plant B. tsuneonis limited citrus[9], so the prediction of the potential distribution of $B$. tsuneonis in China considered the factor of host distribution. Additionally, the irrigation and land use map were also taken into consideration for Carpomya vesuviana[8] and Rhagoletis pomonella[34]. The land use map removed the desert, river and lake, and urban land use type, etc. Therefore, the predictions based on the model and the results discussed above surely have some limitations; these additional factors should also be considered, and more detailed risk assessments should be useful for obtaining more scientific predictions.

Acknowledgements. The authors thank Prof. Stauffer at Pennsylvania State University for his valuable suggestions and modifying the draft. We would like to thank Yan Fan, Zhimei Li and all other members of the Plant Quarantine Laboratory of China Agricultural University (CAUPQL); Also we would like to thank Dezhi Li and other members of Key Laboratory for Biological Control of Ministry of Agriculture, Institute of Plant Protection, Chinese Academy of Agricultural Sciences.

\section{References}

1. Chen, Y.L., Zhang, D.E.: Historical evidence for population dynamics of Tibetan migratory locust and the forecast of its outbreak. Insect Science 6, 135-145 (1999) 
2. Feng, C.H., Shan, X.N., Guo, C., Luo, L.M.: The change of potential distribution of Locusta migratoria tibetensis Chen (Orthoptera: Acrididae) from 1961 to 2005. Acta Ent. Sin. 54, 694-700 (2011) (in Chinese)

3. Chen, Y.L.: A new subspecies of Locusta migratoria-Tibetan migratory locust (Locusta migratoria tibetensis subsp. n.). Acta Ent. Sin. 12, 463-475 (1963) (in Chinese)

4. Worner, S.P.: Ecoclimatic assessment of potential establishment of exotic pests. J. Econ. Ent. 81, 973-983 (1988)

5. Vera, M.T., Rodriguez, R., Segura, D.F., Cladera, J.L., Sutherst, R.W.: Potential geographical distribution of the Mediterranean fruit fly, Ceratitis capitata (Diptera:Tephritidae), with emphasis on Argentina and Australia. Environ. Ent. 31, 1009-1022 (2002)

6. Yonow, T., Sutherst, R.W.: The geographical distribution of the Queensland fruit fly, Bactrocera tryoni, in relation to climate. Aus. J. Agr. Res. 49, 935-953 (1998)

7. Sutherst, R.W., Collyer, B.S., Yonow, T.: The vulnerability of Australian horticulture to the Queensland fruit fly, Bactrocera tryoni, under climate change. Aus. J. Agr. Res. 51, 467-480 (2000)

8. Lv, W.G., Lin, W., Li, Z.H., Geng, J., Wan, F.H., Wang, Z.L.: Potential geographic distribution of Ber fruit fly, Carpomya vesuviana Costa, in China. Plant Quarantine 6, 343-347 (2008) (in Chinese)

9. Wang, J.W., Li, Z.H., Chen, H.J., Geng, J., Wang, Z.L., Wan, F.H.: The potential geographic distribution of Bactrocera tsuneonis (Diptera: Tephritidae). Plant Quarantine 1, 1-4 (2009) (in Chinese)

10. Ni, W.L., Li, Z.H., Wan, F.H., Wang, Z.L.: Potential geographic distribution of Bactrocera scutellata (Hendel), in China. Chin. J. Biol. Control (add) 2, 61-67 (2009) (in Chinese)

11. Qu, W.W., Li, Z.H., Wan, F.H., Wang, Z.L.: Potential geographic distribution of papaya fruit fly, in China. Chin. J. Biol. Control (add) 2, 68-73 (2009) (in Chinese)

12. Ni, W.L., Li, Z.H., Chen, H.J., Wan, F.H., Qu, W.W., Zhang, Z., Kriticos, D.J.: Including climate change in pest risk assessment: the peach fruit fly, Bactrocera zonata (Diptera: Tephritidae). Bull. Ent. Res. 10, 1-11 (2011)

13. Olfert, O., Weiss, R.M., Kriticos, D.: Application of general circulation models to assess the potential impact of climate change on potential distribution and relative abundance of Melanoplus sanguinipes (Fabricius) (Orthoptera: Acrididae) in north American. Psyche, 1-9 (2011)

14. Kriticos, D.J.: The role of modelling in weed management. In: Shepherd, R.C.H. (ed.) Proceedings of the Eleventh Australian Weeds Conference, Melbourne, Australia, September 30-October 3, pp. 560-569. Weed Science Society of Victoria (1996)

15. Kriticos, D.J., Sutherst, R.W., Brown, J.R., Adkins, S.A., Maywald, G.F.: Climate change and biotic invasions: a case history of a tropical woody vine. Biol. Invasions 5, 145-165 (2003a)

16. Kriticos, D.J., Sutherst, R.W., Brown, J.R., Adkins, S.A., Maywald, G.F.: Climate change and the potential distribution of an invasive alien plant: Acacia nilotica ssp. indica in Australia. J. Appl. Ecol. 40, 111-124 (2003b)

17. Stephens, A.E.A., Kriticos, D.J., Leriche, A.: The current and future potential geographical distribution of the oriental fruit fly, Bactrocera dorsalis (Diptera: Tephritidae). Bull. Ent. Res. 97, 369-378 (2007)

18. Watt, M.S., Kriticos, D.J., Manning, L.K.: The current and future potential distribution of Melaleuca quinquenervia. Eur. Weed Research Society Weed Research 49, 381-390 (2009) 
19. Sutherst, R.W., Maywald, G.F., Kriticos, D.J.: CLIMEX Version 3: User's Guide. Hearne Scientific Software Pty Ltd., http: / /www. Hearne.com. au (accessed November 12, 2008)

20. Sutherst, R.W., Maywald, G.F., Bottomley, W., Bourne, A.: CLIMEX v2 CD and User's Guide. Hearne Scientific Software Pty Ltd., Melbourne (2004)

21. Sutherst, R.W., Maywald, G.F.: A climate model of the red imported fire ant, Solenopsis invicta Buren (Hymenoptera:Formicidae): implications for invasion of new regions, particularly Oceania. Environ. Ent. 34, 317-335 (2005)

22. Kriticos, D.J., Alexander, N.S., Kolomeitz, S.M.: Predicting the potential geographic distribution of weeds in 2080. In: Preston, C., Watts, J.H., Crossman, N.P. (eds.) Proceedings of the Fifteenth Australian Weeds Conference, Adelaide, September 24-28, pp. 27-34. Weed Science Society of Victoria (2006)

23. Qin, D.H., Chen, Y.Y.: Climate and environment evolution in China. Science Press, Beijing (in Chinese)

24. Li, Q., Feng, C.H., Zhang, M., Jiang, F., Yang, G., Luo, L.M.: The biological characteristics of Locusta migratoria tibetensis (Chen). Chin. Bull. Ent. 44, 210-213 (2007) (in Chinese)

25. Jia, M.: The research about the life habit, classes and biological features of locust at Ali Prefecture of TAR. Tibet Agr. Tech. 29, 11-16 (2007) (in Chinese)

26. Guo, F., Chen, Y.L., Lu, B.L.: The biology of the migratory locusts in China. Shandong Science and Technology Press, Shandong (1991) (in Chinese)

27. Chen, Y.L.: The main locust and ecological management of locust plague in China, pp. 68-70. Science Press, Beijing (2007) (in Chinese)

28. Zhang, D.X., Yan, L.N., Kang, L., Ji, Y.J.: Some unorthodox views on the classification and evolution of the migratory locusts in China prompted by molecular population genetic study. Acta Zool. Sin. 49, 675-681 (2003) (in Chinese)

29. Zhang, D.X., Yan, L.N., Kang, L., Ji, Y.J., Hewitt, G.M., Huang, Z.S.: Unexpected relationships of substructured populations in Chinese Locusta migratoria. BMC Evol. Biol. 9, 144-155 (2009)

30. Huang, F.S., Huang, C.M., Liu, J.P.: Studies on the acridoid fauna of Xizang and it's evolution. Acta Ent. Sin. 3, 157-170 (1981) (in Chinese)

31. Chen, Y.L.: A new record of the Desert locust, Schistocerca Gregaria (Forskal) in Tibet, China. Acta Ent. Sin. 25, 67 (1982) (in Chinese)

32. Ye, Q., Huang, X.J.: World mango production and trade. Chin. J. Trop. Agr. 22, 44-49 (2002)

33. Baker, R.H.A., Sansford, C.E., Jarvis, C.H., Cannon, R.J.C., MacLeod, A., Walters, K.F.A.: The role of climate mapping in predicting the potential geographical distribution of non-indigenous pests under current and future climates. Agr. Ecosyst. Environ. 82, 57-71 (2000)

34. Geng, J., Li, Z.H., Rajotte, E.G., Wan, F.H., Lu, X.Y., Wang, Z.L.: Potential geographical distribution of Rhagoletis pomonella (Diptera: Tephritidae) in China. Insect Science, 1-8 (2011) 